\title{
ASYMPTOTIC APPROXIMATIONS TO THE BIAS AND VARIANCE OF A KERNEL-TYPE ESTIMATOR OF THE INTENSITY OF THE CYCLIC POISSON PROCESS WITH THE LINEAR TREND
}

\author{
I WAyAn MANGKu ${ }^{1}$, Siswadi $^{2}$ And Retno Budiarti ${ }^{3}$ \\ ${ }^{1}$ Department of Mathematics, Bogor Agricultural University, Jln. Meranti \\ Kampus IPB Darmaga, Bogor 16680, Indonesia, wayan.mangku@gmail.com \\ ${ }^{2}$ Department of Mathematics, Bogor Agricultural University, Jln. Meranti \\ Kampus IPB Darmaga, Bogor 16680, Indonesia, siswadi@inrr.org \\ ${ }^{3}$ Department of Mathematics, Bogor Agricultural University, Jln. Meranti \\ Kampus IPB Darmaga, Bogor 16680, Indonesia
}

\begin{abstract}
From the previous research, a kernel-type estimator of the intensity of the cyclic Poisson process with the linear trend has been constructed using a single realization of the Poisson process observed in a bounded interval. This proposed estimator has been proved to be consistent as the size of the observation interval tends to infinity. In this paper, asymptotic approximations to its bias, variance and MSE (Mean-Squared-Error) are computed. Asymptotically optimal bandwidth is also derived.

Key words: Cyclic Poisson process, intensity function, linear trend, asymptotic bias, asymptotic variance.
\end{abstract}

\begin{abstract}
Abstrak. Pada penelitian sebelumnya telah dirumuskan suatu penduga tipe kernel bagi fungsi intensitas proses Poisson siklik dengan tren linear, yang hanya menggunakan realisasi tunggal proses Poisson yang diamati pada interval terbatas. Kemudian peneliti sebelumnya juga telah membuktikan kekonsistenan penduga ini, jika ukuran interval pengamatannya menuju takhingga. Pada paper ini ditentukan pendekatan asimtotik untuk bias, ragam dan MSE (Mean-Squared-Error) penduga tersebut. Pilihan bandwidth optimal asimtotik juga diberikan.
\end{abstract}

Kata kunci: Proses Poisson siklik, fungsi intensitas, tren linear, bias asimtotik, ragam asimtotik.

2000 Mathematics Subject Classification: 62E20, 62G20, 62M20. Received: 17-12-2010, revised: 11-02-2011, accepted: 13-02-2011. 


\section{Introduction}

We consider a Poisson process $N$ in $[0, \infty)$ with (unknown) intensity function $\lambda$, which is assumed to be locally integrable and consists of two components, namely a periodic (cyclic) component with period $\tau>0$ and a linear trend component. Hence, for each $s \in[0, \infty)$, the intensity function $\lambda$ can be written as

$$
\lambda(s)=\lambda_{c}(s)+a s,
$$

where $\lambda_{c}(s)$ is a periodic function with period $\tau$ and $a$ is the slope of the linear trend. We do not assume any parametric form of $\lambda_{c}$ except it is periodic, that is the equation

$$
\lambda_{c}(s+k \tau)=\lambda_{c}(s)
$$

holds true for all $s \in[0, \infty)$ and all $k \in \mathbb{Z}$, where $\mathbb{Z}$ denotes the set of integers. We consider a Poisson process in $[0, \infty)$ instead of the one in $\mathbb{R}$, due to the intensity function $\lambda$ has to satisfy (1) and must be nonnegative. By a similar reason, we restrict our attention to the case $a>0$. Throughout we also assume that the period $\tau$ is known, but the slope $a$ and the function $\lambda_{c}$ on $[0, \tau)$ are both unknown.

Suppose that, for some $\omega \in \Omega$, a single realization $N(\omega)$ of the Poisson process $N$ defined on a probability space $(\Omega, \mathcal{F}, \mathbb{P})$, with intensity function $\lambda$ (cf. (1)) is observed in an interval $[0, n]$.

A consistent kernel-type estimator of $\lambda_{c}$ at a given point $s \in[0, \infty)$ using a single realization $N(\omega)$ of the Poisson process $N$ observed in interval $[0, n]$ has been constructed in [5]. Our aim in this paper is to compute asymptotic approximations to the bias, variance and MSE (mean-squared error) of this estimator.

Throughout this paper we assume that $s$ is a Lebesgue point of $\lambda$, that is we have $\lim _{h \downarrow 0} \frac{1}{2 h} \int_{-h}^{h}|\lambda(s+x)-\lambda(s)| d x=0$ (e.g. see [6], pp. 107-108), which automatically means that $s$ is a Lebesgue point of $\lambda_{c}$ as well.

Since $\lambda_{c}$ is a periodic function with period $\tau$, the problem of estimating $\lambda_{c}$ at a given point $s \in[0, \infty)$ can be reduced to the problem of estimating $\lambda_{c}$ at a given point $s \in[0, \tau)$. Therefore, thoughout we assume that $s \in[0, \tau)$.

The rest of the paper is organized as follows. Our main results are presented in section 2. The proofs of these main results are presented in section 3 and section 4.

\section{Main Results}

Suppose that $K: \mathbb{R} \rightarrow \mathbb{R}$ is a function, called kernel, which satisfies the following conditions: (K1) $K$ is a probability density function, (K2) $K$ is bounded, and (K3) $K$ has support in $[-1,1]$. Let also $h_{n}$, which is called bandwidth, be a sequence of positive real numbers converging to 0 , that is $h_{n} \downarrow 0$ as $n \rightarrow \infty$.

An estimator of the slope $a$ has been proposed in [1], which is given by

$$
\hat{a}_{n}=\frac{2 N([0, n])}{n^{2}} \text {. }
$$


The estimator of $\lambda_{c}$ at a given point $s \in[0, \tau)$ constructed in [5], is given by

$$
\hat{\lambda}_{c, n, K}(s)=\frac{1}{\ln n} \sum_{k=1}^{\infty} \frac{1}{k h_{n}} \int_{0}^{n} K\left(\frac{x-(s+k \tau)}{h_{n}}\right) N(d x)-\hat{a}_{n}\left(s+\frac{n}{\ln n}\right) .
$$

Note that the estimator given in (4), which is using a general kernel function $K$, is a generalization of the one proposed in [1], which only consider a uniform kernel. A kernel-type estimator of the intensity of a cyclic Poisson process without trend has been proposed and studied in [2] and [3].

In [1], statistical properties of $\hat{a}_{n}$ given in (3) has been proved. We present again these results in the following lemma, due to they are needed in the proofs of our main results.

Lemma 2.1. Suppose that the intensity function $\lambda$ satisfies (1) and is locally integrable. Then we have

$$
\mathbb{E}\left(\hat{a}_{n}\right)=a+\frac{2 \theta}{n}+\mathcal{O}\left(\frac{1}{n^{2}}\right)
$$

and

$$
\operatorname{Var}\left(\hat{a}_{n}\right)=\frac{2 a}{n^{2}}+\mathcal{O}\left(\frac{1}{n^{3}}\right)
$$

as $n \rightarrow \infty$, where $\theta=\tau^{-1} \int_{0}^{\tau} \lambda_{c}(s) d s$, the global intensity of the cyclic component $\lambda_{c}$. MSE of $\hat{a}_{n}$ is given by

$$
\operatorname{MSE}\left(\hat{a}_{n}\right)=\frac{4 \theta^{2}+2 a}{n^{2}}+\mathcal{O}\left(\frac{1}{n^{3}}\right)
$$

as $n \rightarrow \infty$.

Consistency of $\hat{\lambda}_{c, n, K}(s)$ has been established in [5]. Main results of this paper are presented in Theorems 2.2 and 2.3. In the first theorem, an asymptotic approximation to the expectation of the estimator is presented, while an asymptotic approximation to its variance is given in the second theorem.

Theorem 2.2. Suppose that the intensity function $\lambda$ satisfies (1) and is locally integrable. If, in addition, the kernel function $K$ is symmetrical around 0 and satisfies conditions $(K 1),(K 2),(K 3), h_{n} \downarrow 0, h_{n}^{2} \ln n \rightarrow \infty$, and $\lambda_{c}$ has finite second derivative $\lambda_{c}^{\prime \prime}$ at $s$, then

$$
\mathbb{E} \hat{\lambda}_{c, n, K}(s)=\lambda_{c}(s)+\frac{\lambda_{c}^{\prime \prime}(s)}{2} h_{n}^{2} \int_{-1}^{1} x^{2} K(x) d x+o\left(h_{n}^{2}\right),
$$

as $n \rightarrow \infty$. 
Theorem 2.3. Suppose that the intensity function $\lambda$ satisfies (1) and is locally integrable. If, in addition, the kernel function $K$ satisfies conditions $(K 1),(K 2),(K 3)$, $h_{n} \downarrow 0$ and $h_{n} \ln n \rightarrow \infty$, then

$$
\operatorname{Var}\left(\hat{\lambda}_{c, n, K}(s)\right)=\frac{a \tau}{2 h_{n} \ln n} \int_{-1}^{1} K^{2}(x) d x+o\left(\frac{1}{h_{n} \ln n}\right),
$$

as $n \rightarrow \infty$, provided $s$ is a Lebesgue point of $\lambda_{c}$.

We note that, the results presented in Theorems 2.2 and 2.3, are generalization of those results in Theorem 2 of [1]. The results in Theorem 2 of [1] (cf. also Theorems 3.1 and 3.2 of [4]) are only valid for the estimator employing the uniform kernel, while the results presented in Theorems 2.2 and 2.3 are valid for any kernel function $K$, provided it satisfies conditions $(K 1),(K 2)$ and $(K 3)$.

By (7) and (8) of Theorems 2.2 and 2.3, one can obtain an asymptotic approximation to the MSE of $\hat{\lambda}_{c, n, K}(s)$ as follows

$$
\begin{aligned}
\operatorname{MSE}\left(\hat{\lambda}_{c, n, K}(s)\right)= & \frac{a \tau}{2 h_{n} \ln n} \int_{-1}^{1} K^{2}(x) d x+\frac{1}{4}\left(\lambda_{c}^{\prime \prime}(s) \int_{-1}^{1} x^{2} K(x) d x\right)^{2} h_{n}^{4} \\
& +o\left(\frac{1}{h_{n} \ln n}\right)+o\left(h_{n}^{4}\right),
\end{aligned}
$$

as $n \rightarrow \infty$. Now we consider the r.h.s. of (9). By minimizing the sum of its first and second terms, one can obtain an asymptotically optimal bandwidth, which is given by

$$
h_{n}=\left(\frac{a \tau \int_{-1}^{1} K^{2}(x) d x}{2\left(\lambda_{c}^{\prime \prime}(s) \int_{-1}^{1} x^{2} K(x) d x\right)^{2}}\right)^{\frac{1}{5}}(\ln n)^{-\frac{1}{5}} .
$$

If this optimal bandwidth is used, then the MSE of $\hat{\lambda}_{c, n, K}(s)$ will converge to 0 of rate $\mathcal{O}\left((\ln n)^{-4 / 5}\right)$, as $n \rightarrow \infty$.

\section{Proof of Theorem $\mathbf{2 . 2}$}

Expectation of $\hat{\lambda}_{c, n, K}(s)$ can be computed as follows

$$
\mathbb{E} \hat{\lambda}_{c, n, K}(s)=\frac{1}{\ln n} \sum_{k=1}^{\infty} \frac{1}{k h_{n}} \int_{0}^{n} K\left(\frac{x-(s+k \tau)}{h_{n}}\right) \mathbb{E} N(d x)-\left(s+\frac{n}{\ln n}\right) \mathbb{E} \hat{a}_{n} .
$$

First we consider the first term on the r.h.s. of (10). This term can be written as

$$
\begin{aligned}
& \frac{1}{\ln n} \sum_{k=1}^{\infty} \frac{1}{k h_{n}} \int_{0}^{n} K\left(\frac{x-(s+k \tau)}{h_{n}}\right) \lambda(x) d x \\
= & \frac{1}{\ln n} \sum_{k=1}^{\infty} \frac{1}{k h_{n}} \int_{\mathbb{R}} K\left(\frac{x-(s+k \tau)}{h_{n}}\right) \lambda(x) I(x \in[0, n]) d x,
\end{aligned}
$$


where $I$ denotes the indicator function. By a change of variable and using (2), the r.h.s. of (11) can be written as

$$
\begin{aligned}
& \frac{1}{\ln n} \sum_{k=1}^{\infty} \frac{1}{k h_{n}} \int_{\mathbb{R}} K\left(\frac{x}{h_{n}}\right) \lambda(x+s+k \tau) I(x+s+k \tau \in[0, n]) d x \\
= & \frac{1}{\ln n} \sum_{k=1}^{\infty} \frac{1}{k h_{n}} \int_{\mathbb{R}} K\left(\frac{x}{h_{n}}\right) \lambda_{c}(x+s) I(x+s+k \tau \in[0, n]) d x \\
& +\frac{1}{\ln n} \sum_{k=1}^{\infty} \frac{1}{k h_{n}} \int_{\mathbb{R}} K\left(\frac{x}{h_{n}}\right) a(x+s+k \tau) I(x+s+k \tau \in[0, n]) d x .
\end{aligned}
$$

The first term on the r.h.s. of (12) can be written as

$$
\frac{1}{h_{n} \ln n} \int_{\mathbb{R}} K\left(\frac{x}{h_{n}}\right) \lambda_{c}(x+s) \sum_{k=1}^{\infty} \frac{1}{k} I(x+s+k \tau \in[0, n]) d x .
$$

By a change of variable, the quantity in (13) can be expressed as

$$
\frac{1}{\ln n} \int_{-1}^{1} K(x) \lambda_{c}\left(s+x h_{n}\right) \sum_{k=1}^{\infty} \frac{1}{k} I\left(x h_{n}+s+k \tau \in[0, n]\right) d x .
$$

Since

$$
\sum_{k=1}^{\infty} \frac{1}{k} I\left(x h_{n}+s+k \tau \in[0, n]\right) d x=\ln n+\mathcal{O}(1),
$$

as $n \rightarrow \infty$, the quantity in (14) can be written as

$$
\begin{aligned}
& \frac{1}{\ln n} \int_{-1}^{1} K(x) \lambda_{c}\left(s+x h_{n}\right)(\ln n+\mathcal{O}(1)) d x \\
& =\int_{-1}^{1} K(x) \lambda_{c}\left(s+x h_{n}\right) d x+\mathcal{O}\left(\frac{1}{\ln n}\right),
\end{aligned}
$$

as $n \rightarrow \infty$. By a Taylor expansion, $\lambda_{c}\left(s+x h_{n}\right)$ can be written as

$$
\lambda_{c}\left(s+x h_{n}\right)=\lambda_{c}(s)+\frac{\lambda_{c}^{\prime}(s)}{1 !} x h_{n}+\frac{\lambda_{c}^{\prime \prime}(s)}{2 !} x^{2} h_{n}^{2}+o\left(h_{n}^{2}\right),
$$

as $n \rightarrow \infty$. Substituting (17) to the r.h.s. of (16), we obtain

$$
\lambda_{c}(s) \int_{-1}^{1} K(x) d x+\lambda_{c}^{\prime}(s) h_{n} \int_{-1}^{1} x K(x) d x+\frac{\lambda_{c}^{\prime \prime}(s)}{2} h_{n}^{2} \int_{-1}^{1} x^{2} K(x) d x+o\left(h_{n}^{2}\right),
$$

as $n \rightarrow \infty$. Since $\int_{-1}^{1} K(x) d x=1$ and the second term of (18) is equal to zero, we find the first term on the r.h.s. of (12) is equal to

$$
\lambda_{c}(s)+\frac{\lambda_{c}^{\prime \prime}(s)}{2} h_{n}^{2} \int_{-1}^{1} x^{2} K(x) d x+o\left(h_{n}^{2}\right),
$$

as $n \rightarrow \infty$ 
Next we consider the second term on the r.h.s. of (12). This term can be written as

$$
\begin{aligned}
& \frac{a}{h_{n} \ln n} \int_{\mathbb{R}} K\left(\frac{x}{h_{n}}\right) x \sum_{k=1}^{\infty} \frac{1}{k} I(x+s+k \tau \in[0, n]) d x \\
& +\frac{a s}{h_{n} \ln n} \int_{\mathbb{R}} K\left(\frac{x}{h_{n}}\right) \sum_{k=1}^{\infty} \frac{1}{k} I(x+s+k \tau \in[0, n]) d x \\
& +\frac{a \tau}{h_{n} \ln n} \int_{\mathbb{R}} K\left(\frac{x}{h_{n}}\right) \sum_{k=1}^{\infty} I(x+s+k \tau \in[0, n]) d x .
\end{aligned}
$$

By (15), the quantity in (20) can be written as

$$
\begin{aligned}
& \frac{a}{h_{n} \ln n}(\ln n+\mathcal{O}(1)) \int_{\mathbb{R}} K\left(\frac{x}{h_{n}}\right) x d x \\
& +\frac{a s}{h_{n} \ln n}(\ln n+\mathcal{O}(1)) \int_{\mathbb{R}} K\left(\frac{x}{h_{n}}\right) d x \\
& +\frac{a \tau}{h_{n} \ln n}\left(\frac{n}{\tau}+\mathcal{O}(1)\right) \int_{\mathbb{R}} K\left(\frac{x}{h_{n}}\right) d x .
\end{aligned}
$$

Since the kernel $K$ is bounded and $\int_{-1}^{1} x d x=0$, the first term of (21) is equal to zero. A simple calculation shows that the second term of (21) is equal to

$$
\frac{a s}{h_{n} \ln n}(\ln n+\mathcal{O}(1)) \int_{-1}^{1} K(x) d x h_{n}=a s+\mathcal{O}\left(\frac{1}{\ln n}\right),
$$

as $n \rightarrow \infty$. The third term of $(21)$ is equal to $a n /(\ln n)+\mathcal{O}\left((\ln n)^{-1}\right)$ as $n \rightarrow \infty$. Hence, the second term on the r.h.s. of (12) is equal to

$$
a s+\frac{a n}{\ln n}+\mathcal{O}\left(\frac{1}{\ln n}\right),
$$

as $n \rightarrow \infty$. Combining (19) and (22), we obtain that the first term on the r.h.s. of (10) is equal to

$$
\lambda_{c}(s)+\frac{\lambda_{c}^{\prime \prime}(s)}{2} h_{n}^{2} \int_{-1}^{1} x^{2} K(x) d x+a s+\frac{a n}{\ln n}+o\left(h_{n}^{2}\right)+\mathcal{O}\left(\frac{1}{\ln n}\right),
$$

as $n \rightarrow \infty$.

Now we consider the second term on the r.h.s. of (10). By (5) of Lemma 2.1, this quantity can be computed as follows

$$
\left(s+\frac{n}{\ln n}\right)\left(a+\frac{2 \theta}{n}+\mathcal{O}\left(\frac{1}{n^{2}}\right)\right)=a s+\frac{a n}{\ln n}+\mathcal{O}\left(\frac{1}{\ln n}\right),
$$

as $n \rightarrow \infty$. By the assumption $h_{n}^{2} \ln n \rightarrow \infty$, we have $\mathcal{O}\left((\ln n)^{-1}\right)=o\left(h_{n}^{2}\right)$, as $n \rightarrow \infty$. Combining the results in (23) and (24) we obtain

$$
\mathbb{E} \hat{\lambda}_{c, n, K}(s)=\lambda_{c}(s)+\frac{\lambda_{c}^{\prime \prime}(s)}{2} h_{n}^{2} \int_{-1}^{1} x^{2} K(x) d x+o\left(h_{n}^{2}\right),
$$

as $n \rightarrow \infty$. This completes the proof of Theorem 2.2. 


\section{Proof of Theorem 2.3}

Variance of $\hat{\lambda}_{c, n, K}(s)$ can be computed as follows

$$
\begin{aligned}
& \operatorname{Var}\left(\hat{\lambda}_{c, n, K}(s)\right) \\
& =\operatorname{Var}\left(\frac{1}{\ln n} \sum_{k=1}^{\infty} \frac{1}{k h_{n}} \int_{0}^{n} K\left(\frac{x-(s+k \tau)}{h_{n}}\right) N(d x)\right) \\
& +\operatorname{Var}\left(\hat{a}_{n}\left(s+\frac{n}{\ln n}\right)\right) \\
& -2 \operatorname{Cov}\left(\frac{1}{\ln n} \sum_{k=1}^{\infty} \frac{1}{k h_{n}} \int_{0}^{n} K\left(\frac{x-(s+k \tau)}{h_{n}}\right) N(d x), \hat{a}_{n}\left(s+\frac{n}{\ln n}\right)\right) .
\end{aligned}
$$

First, we consider the first term on the r.h.s. of (25). Since $h_{n} \downarrow 0$ as $n \rightarrow \infty$, then for sufficiently large $n$, interval $\left[s+k \tau-h_{n}, s+k \tau+h_{n}\right]$ and $\left[s+j \tau-h_{n}, s+\right.$ $\left.j \tau+h_{n}\right]$ are disjoint for all $k \neq j$. This implies, for all $k \neq j$,

$$
K\left(\frac{x-(s+k \tau)}{h_{n}}\right) N(d x) \text { and } K\left(\frac{x-(s+j \tau)}{h_{n}}\right) N(d x)
$$

are independent. Hence, the variance in the first term on the r.h.s. of (25) can be computed as follows

$$
\begin{aligned}
& \frac{1}{\left(h_{n} \ln n\right)^{2}} \sum_{k=1}^{\infty} \frac{1}{k^{2}} \int_{0}^{n} K^{2}\left(\frac{x-(s+k \tau)}{h_{n}}\right) \operatorname{Var}(N(d x)) \\
= & \frac{1}{\left(h_{n} \ln n\right)^{2}} \sum_{k=1}^{\infty} \frac{1}{k^{2}} \int_{0}^{n} K^{2}\left(\frac{x-(s+k \tau)}{h_{n}}\right) \mathbb{E} N(d x) \\
= & \frac{1}{\left(h_{n} \ln n\right)^{2}} \sum_{k=1}^{\infty} \frac{1}{k^{2}} \int_{0}^{n} K^{2}\left(\frac{x-(s+k \tau)}{h_{n}}\right) \lambda(x) d x .
\end{aligned}
$$

By a change of variable and using (2), the r.h.s. of (26) can be written as

$$
\begin{aligned}
& \frac{1}{\left(h_{n} \ln n\right)^{2}} \sum_{k=1}^{\infty} \frac{1}{k^{2}} \int_{\mathbb{R}} K^{2}\left(\frac{x}{h_{n}}\right) \lambda(x+s+k \tau) I(x+s+k \tau \in[0, n]) d x \\
= & \frac{1}{\left(h_{n} \ln n\right)^{2}} \sum_{k=1}^{\infty} \frac{1}{k^{2}} \int_{\mathbb{R}} K^{2}\left(\frac{x}{h_{n}}\right) \lambda_{c}(x+s) I(x+s+k \tau \in[0, n]) d x \\
& +\frac{1}{\left(h_{n} \ln n\right)^{2}} \sum_{k=1}^{\infty} \frac{1}{k^{2}} \int_{\mathbb{R}} K^{2}\left(\frac{x}{h_{n}}\right) a(x+s+k \tau) I(x+s+k \tau \in[0, n]) d x .
\end{aligned}
$$


The first term on the r.h.s. of (27) can be written as

$$
\begin{aligned}
& \frac{1}{\left(h_{n} \ln n\right)^{2}} \int_{\mathbb{R}} K^{2}\left(\frac{x}{h_{n}}\right)\left(\lambda_{c}(x+s)-\lambda_{c}(s)\right) \sum_{k=1}^{\infty} \frac{1}{k^{2}} I(x+s+k \tau \in[0, n]) d x \\
& +\frac{\lambda_{c}(s)}{\left(h_{n} \ln n\right)^{2}} \int_{\mathbb{R}} K^{2}\left(\frac{x}{h_{n}}\right) \sum_{k=1}^{\infty} \frac{1}{k^{2}} I(x+s+k \tau \in[0, n]) d x .
\end{aligned}
$$

Now we see that

$$
\sum_{k=1}^{\infty} \frac{1}{k^{2}} I(x+s+k \tau \in[0, n]) d x=\mathcal{O}(1),
$$

as $n \rightarrow \infty$. Since $s$ is a Lebesque point of $\lambda_{c}$ then

$$
\frac{1}{2 h_{n}} \int_{-h_{n}}^{h_{n}}\left(\lambda_{c}(x+s)-\lambda_{c}(s)\right)=o(1),
$$

as $n \rightarrow \infty$. Since the kernel function $K$ is bounded and having support $[-1,1]$, by (29) and (30), we obtain that the first term on the r.h.s. of (28) is equal to $\left.o\left((\ln n)^{-2}\left(h_{n}\right)^{-1}\right)\right)=o\left(\left(h_{n} \ln n\right)^{-1}\right)$, as $n \rightarrow \infty$. By a similar argument, the second term on the r.h.s. of (28) is equal to $\mathcal{O}\left(1 /\left(h_{n} \ln n\right)^{2}\right)=o\left(\left(h_{n} \ln n\right)^{-1}\right)$, as $n \rightarrow \infty$. This last result due to the assumption $h_{n} \ln n \rightarrow \infty$ as $n \rightarrow \infty$.

Next we consider the second term on the r.h.s. of (27). This term can be written as

$$
\begin{aligned}
& \frac{a}{\left(h_{n} \ln n\right)^{2}} \int_{\mathbb{R}} K^{2}\left(\frac{x}{h_{n}}\right)(x+s) \sum_{k=1}^{\infty} \frac{1}{k^{2}} I(x+s+k \tau \in[0, n]) d x \\
& +\frac{a \tau}{\left(h_{n} \ln n\right)^{2}} \int_{\mathbb{R}} K^{2}\left(\frac{x}{h_{n}}\right) \sum_{k=1}^{\infty} \frac{1}{k} I(x+s+k \tau \in[0, n]) d x .
\end{aligned}
$$

By (29), the first term of (31) can be simplified as

$$
\begin{aligned}
& \mathcal{O}(1) \frac{a}{\left(h_{n} \ln n\right)^{2}} \int_{\mathbb{R}} K^{2}\left(\frac{x}{h_{n}}\right)(x+s) d x \\
= & \mathcal{O}(1) \frac{a}{(\ln n)^{2} h_{n}} \int_{\mathbb{R}} K^{2}(x)\left(x h_{n}+s\right) d x \\
= & \mathcal{O}\left(\frac{1}{h_{n}(\ln n)^{2}}\right)=o\left(\frac{1}{h_{n} \ln n}\right),
\end{aligned}
$$

as $n \rightarrow \infty$. By a change of variable, the second term of (31) can be expressed as

$$
\begin{aligned}
& \frac{a \tau}{h_{n}(\ln n)^{2}} \int_{\mathbb{R}} K^{2}(x) \sum_{k=1}^{\infty} \frac{1}{k} I\left(x h_{n}+s+k \tau \in[0, n]\right) d x \\
= & \frac{a \tau}{h_{n}(\ln n)^{2}} \int_{\mathbb{R}} K^{2}(x) d x(\ln n+\mathcal{O}(1)) \\
= & \frac{a \tau}{h_{n} \ln n} \int_{-1}^{1} K^{2}(x) d x+o\left(\frac{1}{h_{n} \ln n}\right)
\end{aligned}
$$


as $n \rightarrow \infty$. Hence we have that the first term on the r.h.s. of (25) is equal to

as $n \rightarrow \infty$.

$$
\frac{a \tau}{h_{n} \ln n} \int_{-1}^{1} K^{2}(x) d x+o\left(\frac{1}{h_{n} \ln n}\right)
$$

Next we consider the second term on the r.h.s. of (25). By (6) of Lemma 2.1, this quantity can be computed as follows

$$
\begin{aligned}
\left(s+\frac{n}{\ln n}\right)^{2} \operatorname{Var}\left(\hat{a}_{n}\right) & =\left(s^{2}+\frac{n^{2}}{(\ln n)^{2}}+\frac{2 s n}{\ln n}\right)\left(\frac{2 a}{n^{2}}+\mathcal{O}\left(\frac{1}{n^{3}}\right)\right) \\
& =\mathcal{O}\left(\frac{1}{(\ln n)^{2}}\right)=\mathcal{O}\left(\frac{1}{h_{n} \ln n}\right) \frac{h_{n}}{\ln n} \\
& =o\left(\frac{1}{h_{n} \ln n}\right),
\end{aligned}
$$

as $n \rightarrow \infty$.

Now we consider the third term on the r.h.s. of (25). Since the first term on the r.h.s. of $(25)$ is $\mathcal{O}\left(\left(h_{n} \ln n\right)^{-1}\right)$ and its second term is $o\left(\left(h_{n} \ln n\right)^{-1}\right)$ as $n \rightarrow \infty$, by Cauchy-Schwarz inequality, we have that the third term on the r.h.s. of (25) is of order $o\left(\left(h_{n} \ln n\right)^{-1}\right)$ as $n \rightarrow \infty$. Combining this result with (34) and (35), we have

$$
\operatorname{Var}\left(\hat{\lambda}_{c, n, K}(s)\right)=\frac{a \tau}{h_{n} \ln n} \int_{-1}^{1} K^{2}(x) d x+o\left(\frac{1}{h_{n} \ln n}\right),
$$

as $n \rightarrow \infty$. This completes the proof of Theorem 2.3.

\section{References}

[1] Helmers, R., and Mangku, I W., "Estimating the intensity of a cyclic Poisson process in the presence of linear trend", Annals Inst. of Statistical Mathematics, 61:3 (2009), 599-628.

[2] Helmers, R., Mangku I W., and Zitikis, R., "Consistent estimation of the intensity function of a cyclic Poisson process", Journal of Multivariate Analysis, 84 (2003), 19-39.

[3] Helmers, R., Mangku I W., and Zitikis, R., "Statistical properties of a kernel-type estimator of the intensity function of a cyclic Poisson process", Journal of Multivariate Analysis, 92 (2005), 1-23.

[4] Helmers, R., Mangku I W., and Zitikis, R., "A non-parametric estimator for the doubly periodic Poisson intensity function", Statistical Methodology, 4 (2007), 481-492.

[5] Mangku, I W., Siswadi and Budiarti, R., "Consistency of a kernel-type estimator of the intensity of the cyclic Poisson process with the linear trend", Journal of the Indonesian Mathematical Society, 15:1 (2009), 37-48.

[6] Wheeden, R.L., and Zygmund, A., Measure and Integral: An Introduction to Real Analysis. Marcel Dekker, New York, 1977. 